\title{
Pratiques
}

Linguistique, littérature, didactique

187-188 | 2020

Enseignement du texte littéraire dans l'espace

francophone: pratiques, formation, recherche

\section{Prise en compte du sujet lecteur à l'école élémentaire. Éléments de constat chez les professeurs des écoles stagiaires exerçant en cycle 3}

How the reader subjectivity is taken into account in elementary school.

Observation among trainee teachers practicing in $4^{\text {th }}-5^{\text {th }}$ grades/Years 6-7

Véronique Larrivé

\section{(2) OpenEdition}

Journals

Édition électronique

URL : https://journals.openedition.org/pratiques/9362

DOI : $10.4000 /$ pratiques.9362

ISSN : 2425-2042

Éditeur

Centre de recherche sur les médiations (CREM)

Référence électronique

Véronique Larrivé, «Prise en compte du sujet lecteur à l'école élémentaire. Éléments de constat chez les professeurs des écoles stagiaires exerçant en cycle 3 », Pratiques [En ligne], 187-188| 2020, mis en ligne le 12 décembre 2020, consulté le 21 juillet 2021. URL : http://journals.openedition.org/pratiques/ 9362 ; DOI : https://doi.org/10.4000/pratiques.9362

Ce document a été généré automatiquement le 21 juillet 2021.

(c) Tous droits réservés 


\section{Prise en compte du sujet lecteur à l'école élémentaire. Éléments de constat chez les professeurs des écoles stagiaires exerçant en cycle 3}

How the reader subjectivity is taken into account in elementary school. Observation among trainee teachers practicing in $4^{\text {th }}-5^{\text {th }}$ grades/Years 6-7

Véronique Larrivé

\section{Prise en compte du sujet lecteur à l'école élémentaire. Éléments de constat chez les professeurs des écoles stagiaires exerçant en cycle 3}

1 À la marge de l'interrogation sur la lecture analytique dans le secondaire, notre article concerne les pratiques effectives d'étude des textes dans l'enseignement de la lecture littéraire au cycle 3 de l'école primaire ( $4^{\mathrm{e}}$ et $5^{\mathrm{e}}$ années de l'école élémentaire, enfants de 8 à 10 ans), tel qu'il est mis en œuvre par les professeurs d'école stagiaires (PES), qui, depuis septembre 2014, effectuent leur année de stage en alternance entre la formation à l'Éspé ${ }^{1}$ et la responsabilité d'une classe à mi-temps.

Depuis l'avènement, dans les programmes de 2002 pour l'école primaire, de la littérature de jeunesse comme objet d'enseignement dans la discipline du français, un changement s'est opéré dans les prescriptions institutionnelles pour le premier degré, concernant la représentation de la lecture. L'acte de lire n'est officiellement plus réduit à la compréhension des textes mais intègre la dimension subjective de la réception des œuvres littéraires. Dans les programmes de 2015 pour le cycle 3, comme dans ceux de 2018 qui les remplacent pour le français, on trouve ainsi, dans la section «Lecture et compréhension de l'écrit », une rubrique explicitement dédiée aux œuvres littéraires, intitulée «Comprendre un texte littéraire et l'interpréter» $\left(\mathrm{MEN}^{2}, 2015\right)$ puis 
«Comprendre un texte littéraire et se l'approprier » (MEN, 2018). Parmi les « exemples de situations, d'activités et d'outils pour l'élève » proposés dans ces rubriques figurent le partage des impressions, le débat interprétatif et le carnet de lecture, autant de réminiscences de suggestions faites dès 2002 par le ministère de l'Éducation nationale pour une approche de la littérature respectueuse de la sensibilité des élèves et de la singularité de leurs diverses interprétations.

3 Nous proposons d'observer comment les professeurs d'école stagiaires, issus de cursus universitaires présentant une grande diversité, intègrent ces activités et outils dans leur classe. Nous faisons en effet l'hypothèse que leur représentation de l'activité de lecture et de ses enjeux est susceptible d'engendrer des résistances quant à la prise en compte du sujet lecteur dans leurs pratiques de classe. Nous nous demanderons donc si les professeurs d'école stagiaires perçoivent les spécificités de la réception littéraire et ce qu'ils en font dans leur enseignement de la littérature. Où les résistances se situentelles s'il y en a et quelle peut en être la cause ? Pour répondre à ces questions, nous utiliserons un large échantillon de rapports de visite, rédigés par des formateurs Éspé toulousains en 2015-2016 et 2016-2017, rendant compte de l'observation de séances d'enseignement en littérature en cours moyen. Pour compléter ces données, nous avons, en mars 2019, effectué un sondage auprès de tous les PES de l'académie de Toulouse concernant leurs représentations de l'enseignement de la littérature à l'école. Nous examinerons ici les réponses données par les stagiaires exerçant en cycle 3.

4 Nous présenterons d'abord le cadre conceptuel qui sous-tend les démarches prônées en didactique de la littérature, c'est-à-dire les axes de réflexion théorique qui, depuis 20 ans, ont fait évoluer le cadre institutionnel de l'enseignement de la littérature. Nous exposerons ensuite les conditions de recueil des données avant de présenter les résultats obtenus, qui montreront quelles tendances caractérisent l'intégration, par les professeurs des écoles stagiaires, des principes didactiques enseignés à l'Éspé, mais aussi quelles orientations cela suggère de suivre pour infléchir la formation offerte aujourd'hui à l'Inspé3.

\section{Le sujet lecteur, de la théorie littéraire aux programmes de l'école}

5 G. Langlade $(2004$, p. 81-91; 2007, p. 71) définit la lecture subjective comme étant une lecture qui affecte le lecteur comme sujet, c'est-à-dire qui lui procure des émotions, lui fait éprouver des sentiments et déclenche chez lui des jugements axiologiques. Il en résulte que le lecteur est appelé sujet lecteur en tant qu'il répond aux sollicitations du texte avec sa subjectivité, ses émotions, ses goûts, ses conceptions, ses représentations, ses souvenirs.

\section{L'action conjointe du lisant et du lectant pour l'appropriation des textes}

6 Si l'on revient aux concepts proposés par les théoriciens de la lecture littéraire pour modéliser l'activité du lecteur, on obtient, avec les travaux de M. Picard (1986) et V. Jouve (1992), un ensemble de quatre concepts qui rendent compte de ce qui est en jeu chez le sujet lisant: le corps, l'imagination, l'intellect et l'inconscient, appelés respectivement liseur, lisant, lectant et lu. De ces instances lectrices mobilisées chez le 
sujet en train de lire, on peut dégager un couple très prolifique dans la recherche en didactique de la littérature, celui du lisant et du lectant, qui permet de montrer la complémentarité de deux positions face au texte.

Le lectant est la part du lecteur qui incarne la distanciation : il observe le texte avec un point de vue extérieur, utilise sa raison pour analyser des faits textuels et ambitionne une certaine objectivité. À contrario, le lisant représente la part du lecteur qui fait fonctionner son imagination, s'implique émotionnellement dans l'univers fictionnel, entre en empathie avec les personnages et revendique un investissement subjectif (Jouve, 1992, p. 81-82 ; Larrivé, 2015, p.162). Ces deux instances lectrices, qui représentent le double mouvement d'implication et de distanciation dans la lecture littéraire, sont constamment en corrélation, mais parfois aussi en tension quand on attribue à l'une d'elles une place hiérarchiquement privilégiée. Si l'enseignement de la littérature dans le secondaire a longtemps valorisé la posture du lectant, favorisant une attitude distanciée et des techniques d'analyse formelle, notamment avec la promotion de la lecture méthodique dans les programmes du lycée dès 1987 puis du collège en 1996, les apports des didacticiens de la littérature ont, depuis 20 ans, infléchi cette tendance en mettant en avant le nécessaire va-et-vient à instaurer entre les deux instances du lisant et du lectant (Dufays, 2005, p. 97) et en prônant la reconnaissance du lisant comme instance lectrice indispensable pour l'activité fictionnalisante du lecteur (Langlade, 2007). Leur attention s'est donc portée sur la manière dont les élèves s'approprient les œuvres littéraires, c'est-à-dire sur le processus singulier par lequel ils les inscrivent dans leur mémoire individuelle et les intègrent à leur réflexion sur le monde et sur les autres (Shawky-Milcent, 2016, p. 4-5). Cet intérêt pour la réception subjective des œuvres lues nécessite de prendre en compte non seulement l'expérience intellectuelle des élèves lecteurs mais aussi leur expérience émotionnelle face au texte.

Dans un article de 2013 où elle décrit la lente prise en compte de l'élève comme sujet lecteur dans l'enseignement de la littérature, A. Rouxel (2013, p. 116-117) rappelle que les objectifs de la recherche en didactique mise en place depuis les années 2000 sont de réintroduire la subjectivité dans la lecture, de l'humaniser et de lui donner sens. Avant d'observer comment les enseignants stagiaires en cycle 3 entendent ces conseils des didacticiens, voyons ce qu'il en est dans les programmes pour l'école, en CM1 et CM2.

\section{La lecture subjective dans les programmes de 2015 et de 2018}

9 Avec les programmes de 2002 et la première apparition de la littérature comme sousdiscipline du français en cycle 3, la spécificité de la lecture littéraire est enfin reconnue dans l'enseignement de l'école primaire. Sous l'impulsion des travaux de C. Tauveron $(1995 ; 1999$; 2002), les démarches proposées intègrent le carnet de lecteur, pour permettre l'expression et la prise en compte de la singularité des lectures, mais aussi le débat interprétatif permettant le partage des impressions et de la réflexion sur les textes, et instaurant la classe en communauté de lecteurs.

Les programmes de 2015 pour la littérature, dont ceux de 2018 sont très proches, reviennent à des principes similaires. C'est ainsi que, dans le domaine 3 intitulé « La formation de la personne et du citoyen ", on insiste sur la lecture sensible des œuvres :

En français, on s'attache à permettre la réception sensible des œuvres littéraires en développant son expression, la formulation de ses opinions, dans des échanges oraux ou en en recueillant les traces écrites dans des carnets de lecture (MEN, 2015, p. 95 ; 2018, p. 5). 
11 Dans les programmes de la discipline « Français », l'accent est mis sur les enjeux de l'enseignement de la littérature en termes de développement personnel de l'élève :

La littérature est également une part essentielle de l'enseignement du français : elle développe l'imagination, enrichit la connaissance du monde et participe à la construction de soi (ibid., 2015, p. $98 ; 2018$, p. 8).

Dans les rubriques « Lecture et compréhension de l'écrit ", là où on évoquait en 2015, la compétence "Comprendre un texte littéraire et l'interpréter » (ibid., 2015, p. 104), il s'agit, en 2018, de "Comprendre un texte littéraire et se l'approprier » (ibid., 2018, p.14). Ce changement terminologique évite le flou conceptuel du terme « interprétation » dénoncé dès 2003 par E. Falardeau (2003, p. 673) et atténue ainsi la complexité sémantique, décrite par S. De Croix et B. Shawky-Milcent dans le Dictionnaire de didactique de la littérature (Brillant-Ranou, N. et al., 2020, p. 48-55), de la relation entre compréhension et interprétation, le terme " appropriation » subsumant en effet les deux opérations dans une vision plus globalisante du rapport aux textes. Il apparait alors que, si les titres des rubriques, dans les programmes scolaires, ne sont pas exactement les mêmes en 2015 et en 2018, la réception subjective des textes lus reçoit toujours une attention particulière, comme le montrent les deux extraits cidessous :

Au CM1 et au CM2, [...] les activités de lecture doivent permettre aux élèves de verbaliser, à l'oral ou à l'écrit, leur réception des textes et des œuvres : reformulation ou paraphrase, mise en relation avec son expérience et ses connaissances, mise en relation avec d'autres lectures ou d'autres œuvres, expression d'émotions, de jugements, à l'égard des personnages notamment (MEN, 2015, p. 108).

Au cycle 3, l'accent est mis sur l'appropriation du texte littéraire par l'élève, en lien avec son expérience, ses lectures, ses connaissances, celles qu'il acquiert dans d'autres disciplines, notamment en histoire (ibid., 2018, p. 8).

Il résulte de cet objectif d'appropriation que certaines activités proposées dans le cadre de la lecture d'un texte littéraire, en 2015 comme en 2018, sont bien au service de l'expression et du partage de la réception des œuvres :

Activités permettant de partager ses impressions de lecture, faire des hypothèses d'interprétation et en débattre, confronter des jugements : débats interprétatifs, cercles de lecture, présentations orales, mises en voix avec justification des choix. [...] En lien avec l'écriture et pour préparer les activités de partage des lectures et d'interprétation: cahiers ou carnets de lecture, affichages littéraires, etc. ${ }^{4}$ (ibid., 2015 , p. 106 ; 2018, p. 14).

14 Ce sont ces situations, activités et supports, témoignant d'une approche sensible et partagée des textes, qui nous serviront de cadre pour observer les comptes rendus de visite.

Notre présentation des programmes serait incomplète si nous ne mentionnions la focalisation très forte des préoccupations ministérielles actuelles sur l'enseignement explicite de la compréhension (Bianco \& Lima, 2017, p.14). On peut en effet se demander si cette polarisation un peu exclusive sur la méthodologie de la compréhension ne joue pas un rôle dans les représentations de la lecture littéraire chez les jeunes enseignants. Avant de présenter le protocole de recherche, rappelons maintenant dans quel contexte de formation il a été mis en place. 


\section{Le contexte de formation initiale des professeurs des écoles stagiaires}

16 À partir de septembre 2014, avec la création des Éspé, la formation initiale des professeurs s'organise avec un stage de pratique professionnelle à mi-temps et un temps de formation à l'Éspé à mi-temps également ${ }^{5}$. Pour les professeurs des écoles stagiaires, l'expérience de l'enseignement de la littérature dépend donc beaucoup du partage des tâches avec l'autre enseignant de la classe ${ }^{6}$.

17 Au-delà de cette question organisationnelle, trois points concernant les connaissances en littérature des professeurs des écoles stagiaires doivent être soulignés. Le premier point concerne le métier lui-même : pour devenir professeur des écoles, il faut pouvoir enseigner toutes les disciplines de l'école ${ }^{7}$ et cette polyvalence est difficile à maitriser. En effet, les étudiants du master $\mathrm{MEEF}^{8} 1^{\text {er }}$ degré, qui représentent entre 70 et $80 \%$ de l'effectif des stagiaires, proviennent de cursus universitaires très divers ${ }^{9}$, ce qui génère une grande hétérogénéité face aux connaissances pluridisciplinaires à acquérir. La littérature n'y fait pas exception. Même si la formation donnée en première année de master s'emploie à interroger les représentations que les étudiants peuvent avoir de l'enseignement de la littérature, pour beaucoup de professeurs d'école stagiaires, les derniers souvenirs de lecture littéraire datent des années où ils étaient lycéens. Le second point tient au recrutement : environ un tiers des lauréats du concours n'ont pas suivi les enseignements de la première année du master MEEF et n'ont donc aucune formation didactique quand ils arrivent à l'Éspé en tant que stagiaires. Enfin, le dernier point concerne le temps consacré à l'enseignement de la littérature en deuxième année de master: en raison du mi-temps de stage en classe et de la polyvalence des enseignements, le nombre d'heures consacrées à la didactique du français est assez limité. Ainsi, à Toulouse, depuis 6 ans, les PES n'ont-ils que 36 heures de formation en didactique du français pendant leur année de master 2, alors même que cette formation doit couvrir le « lire, dire, écrire » sur les 3 cycles de l'école primaire. On imagine bien que la place attribuée à l'enseignement de la littérature est donc très réduite ${ }^{10}$. Ces deux mises au point étant faites, nous allons maintenant présenter les différentes données récoltées.

\section{Données récoltées}

\section{Des comptes rendus de visites avec séance de littérature}

18 Nous avons à notre disposition les comptes rendus (CR) de visites correspondant à 144 PES toulousains, affectés en cycle 3 (CM1 et CM2) dans les années 2015-2016 et 2016-2017 $7^{11}$. Comme les PES reçoivent plusieurs visites dans l'année, cela donne un total de 657 rapports de visite parmi lesquels nous n'avons gardé que ceux qui contenaient une séance d'enseignement en lien avec la lecture et/ou la littérature, ce qui fait un total de 266 rapports concernés. Après ce tri, nous avons éliminé ce qui, dans ces séances, ne constituait pas spécifiquement une séance de littérature, à savoir :

- les temps de " poésie $^{12}$ ", sortes de rituels dédiés uniquement à la récitation d'un poème par un ou plusieurs élèves successifs ;

- les présentations de livres, de type « exposés », faites par un ou plusieurs élèves ; 
- les temps de «lecture offerte ", souvent consacrés, dans l'échantillon de comptes rendus de visites à notre disposition, aux histoires mythologiques, comme Le feuilleton d'Hermès ${ }^{13}$ par exemple ;

- les travaux d'écriture amorcés par la lecture d'une œuvre de littérature mais dont les enjeux concernent l'apprentissage de l'écriture et non celui de la lecture littéraire ;

- les exercices de lecture compréhension ne portant pas sur des textes littéraires ${ }^{14}$, même si la plupart de ces exercices permettent de travailler trois compétences nécessaires en littérature: la représentation mentale du contexte et des personnages d'après les informations données par le texte, la flexibilité mentale ou l'adaptation de la représentation aux nouvelles informations données, et la représentation des états mentaux des personnages.

Une fois ces tris opérés, nous obtenons, comme le montre le tableau ci-dessous, 140 séances de littérature à analyser, soit $21 \%$ des 657 CR établis. Cela signifie qu'un cinquième des observations de classe rapportées comportent une séance de littérature. Les stagiaires peuvent en avoir présenté une ou plusieurs dans l'année à leurs tuteurs, mais 35 d'entre eux n'en ont présenté aucune.

Tableau $1:$ Nombre de comptes rendus de visites avec séances de lecture littéraire parmi les comptes rendus disponibles

\begin{tabular}{|c|c|c|c|c|c|c|c|c|c|c|}
\hline & & \multirow[b]{2}{*}{$\begin{array}{c}\text { PES } \\
\text { visités }\end{array}$} & \multirow[b]{2}{*}{$\begin{array}{l}\text { CR de } \\
\text { visite }\end{array}$} & \multirow{2}{*}{$\begin{array}{c}\text { CR relatant } \\
\text { du travail } \\
\text { en lecture } \\
\text { et/ou } \\
\text { littérature }\end{array}$} & \multicolumn{5}{|c|}{ Temps de lecture et/ou littérature non pris en compte } & \multirow{2}{*}{$\begin{array}{l}\text { CR avec } \\
\text { une séance } \\
\text { de lecture } \\
\text { littéraire }\end{array}$} \\
\hline & & & & & $\begin{array}{l}\text { Moment } \\
\text { "poésie" }\end{array}$ & $\begin{array}{l}\text { Moment } \\
\text { "livres" }\end{array}$ & $\begin{array}{l}\text { Lecture } \\
\text { "offerte" }\end{array}$ & $\begin{array}{c}\text { Écriture } \\
\text { sur } \\
\text { littérature }\end{array}$ & $\begin{array}{c}\text { Lecture } \\
\text { hors } \\
\text { littérature }\end{array}$ & \\
\hline \multirow[t]{3}{*}{ 2015-2016 } & CM1 & 30 & 150 & 63 & 10 & 2 & 6 & 1 & 14 & 28 \\
\hline & CM1-CM2 & 9 & 46 & 13 & 2 & 0 & 0 & 0 & 6 & 5 \\
\hline & CM2 & 31 & 133 & 55 & 7 & 2 & 5 & 5 & 4 & 32 \\
\hline \multirow[t]{3}{*}{ 2016-2017 } & CM1 & 34 & 151 & 63 & 8 & 4 & 9 & 2 & 10 & 30 \\
\hline & CM1-CM2 & 9 & 44 & 17 & 0 & 0 & 0 & 3 & 0 & 14 \\
\hline & CM2 & 31 & 133 & 55 & 2 & 5 & 3 & 9 & 5 & 31 \\
\hline \multicolumn{2}{|r|}{ Total } & 144 & 657 & 266 & 29 & 13 & 23 & 20 & 40 & 140 \\
\hline
\end{tabular}

Il est indispensable d'expliquer également que les CR décrivent, pour la plupart, une demi-journée de classe, ce qui représente deux, voire trois, séances d'enseignement. Chacune des descriptions occupe entre 10 et 30 lignes du document, et comprend les commentaires du formateur. Notons également que les CR sont souvent axés sur un aspect de la séance, la démarche, un dispositif ou une activité, et ne peuvent rendre compte de la séance d'enseignement de manière exhaustive. Par exemple, on connait rarement la teneur des questionnaires de compréhension proposés sur les textes, sauf si une question en particulier a attiré l'attention du formateur et qu'il l'a explicitement notée.

\section{Une enquête menée auprès des PES de l'académie}

21 Pour compléter l'observation des comptes rendus de visite, nous avons proposé, en mars 2019, aux PES de toute l'académie toulousaine (huit départements) un sondage concernant leurs représentations de l'enseignement de la littérature. Nous avons obtenu 200 réponses, parmi lesquelles nous avons choisi d'analyser uniquement celles des PES exerçant en cycle 3. Cela représente 60 réponses. 
Tableau 2 : Nombres de réponses obtenues pour le cycle 3, par site départemental, à l'enquête soumise aux PES de l'académie de Toulouse en mars 2019

\begin{tabular}{|l|l|l|l|l|}
\hline \multicolumn{5}{|l|}{ PES de l'académie de Toulouse en 2018-2019 } \\
\hline \multicolumn{2}{|l|}{ Département } & Site Éspé & Nombre de PES & PES en cycle 3 \\
\hline 31 & Haute-Garonne & Toulouse & 124 & 46 \\
\hline 81 & Tarn & Albi & 16 & 4 \\
\hline 65 & Hautes-Pyrénées & Tarbes & 15 & 1 \\
\hline 82 & Tarn-et-Garonne & Montauban & 13 & 6 \\
\hline 32 & Gers & Auch & 12 & 3 \\
\hline 10 & Ariège & Foix & 10 & 0 \\
\hline 12 & Aveyron & Rodez & 8 & 0 \\
\hline 46 & Lot & Cahors & 5 & 0 \\
\hline 60 & questionnaires de PES récupérés et analysés & 60 \\
\hline
\end{tabular}

Les questions posées aux PES sont des questions ouvertes auxquelles ils ont répondu par écrit, sans qu'aucune proposition de réponse ne leur soit donnée ${ }^{15}$. Il leur était demandé d'achever leurs réponses aux trois premières questions concernant leurs représentations de l'enseignement de la littérature (au recto de la feuille), avant de répondre aux trois suivantes, qui renvoyaient plus directement à la compréhension des instructions officielles (au verso) ${ }^{16}$.

Les réponses des PES aux questions posées dans le sondage ont été classées en fonction de leur proximité sémantique, parfois à partir du repérage de mots clés, ce qui a permis d'établir des catégories. Nous avons ensuite comptabilisé le nombre de réponses obtenues par catégorie et avons transformé le résultat en pourcentage par rapport aux 60 sondages dépouillés. Une réponse pouvant parfois entrer dans plusieurs catégories, on ne s'étonnera pas que la somme des pourcentages obtenus soit supérieure à $100 \%$.

\section{Résultats obtenus}

Dans un premier temps, nous allons analyser les représentations des professeurs stagiaires concernant l'enseignement de la littérature, telles qu'elles apparaissent dans leurs réponses au sondage. Ensuite, nous verrons ce qu'il en est de leurs pratiques de classe et nous reviendrons alors au questionnaire pour conclure sur les difficultés constatées.

\section{Les représentations de la lecture littéraire et de son enseignement.}

Nous proposons d'analyser l'ensemble les réponses à la question Q1, relative aux représentations de l'enseignement de la littérature et à ses enjeux, et aux questions qui interrogent deux concepts liés à notre problématique, celui de l'appropriation des textes (Q6) et celui du sujet lecteur (Q4). 
Notons que, si les questions Q1 et Q6 peuvent renvoyer directement au contenu des programmes actuels et à leur compréhension, en revanche, l'expression «sujet lecteur » de la question Q4 n'apparait pas comme telle dans les programmes 2018. On la trouve cependant mentionnée dans la fiche Éduscol pour le cycle 3 consacrée au carnet de lecteur, qui montre que ce dispositif est un outil au service de l'expression de la subjectivité de l'élève :

Pour l'école primaire et plus encore pour le collège, les carnets de lecteur sont également au cœur de la réflexion des recherches en didactique de la littérature qui $\mathrm{y}$ voient un moyen de donner la priorité à la réception effective des textes par les élèves et de les prendre véritablement en compte comme sujets lecteurs (MEN, 2016).

Voici les réponses obtenues aux trois questions posées, suivies des constats qui résultent de leur observation.

Tableau 3 : Types de réponses obtenues à la question Q1 du sondage

\begin{tabular}{|l|l|c|c|}
\hline \multicolumn{2}{|l|}{ Q1 : Que signifie, selon vous, enseigner la littérature? } \\
\hline A & $\begin{array}{l}\text { Développer la culture littéraire des élèves. Leur donner accès au patrimoine } \\
\text { littéraire. }\end{array}$ & $46 / 60$ & $77 \%$ \\
\hline B & Développer le plaisir de lire & $21 / 60$ & $36 \%$ \\
\hline C & Apprendre à comprendre & $20 / 60$ & $33 \%$ \\
\hline D & $\begin{array}{l}\text { Faire connaitre les genres et leurs caractéristiques. Faire analyser, repérer des } \\
\text { invariants. }\end{array}$ & $17 / 60$ & $29 \%$ \\
\hline E & $\begin{array}{l}\text { Ouvrir l'esprit des élèves, aiguiser leur curiosité, développer leur imaginaire, les } \\
\text { amener à s'interroger }\end{array}$ & $14 / 60$ & $23 \%$ \\
\hline F & Apprendre à interpréter & $9 / 60$ & $15 \%$ \\
\hline
\end{tabular}

La question Q1 portant sur le sens de l'expression «enseigner la littérature » et permettant donc de voir les enjeux assignés à cet enseignement, nous proposons d'expliquer la classification opérée à partir des réponses librement rédigées par les PES. Dans la catégorie A, sont regroupées les réponses relevant des champs lexicaux de la culture, de la diversité et de la découverte, appliqués au monde littéraire. La catégorie B regroupe les réponses qui renvoient sémantiquement au plaisir de la lecture (dont " formation d'un lecteur enthousiaste »). La catégorie D est liée aux mots clefs renvoyant aux genres ("types», " codes») et la catégorie E porte sur les expressions évoquant l'ouverture d'esprit, par l'imaginaire ou la réflexion (« faire le lien avec les questions de société ", « se comprendre et comprendre le monde ", " accepter l'autre ", « développer l'imaginaire et la curiosité des élèves »).

Enfin, nous avons regroupé dans les catégories $\mathrm{C}$ et $\mathrm{F}$ les réponses relatives à l'apprentissage de la compréhension et de l'interprétation. Pour départager les réponses entre ces deux rubriques, nous nous sommes appuyée sur les propositions d'É. Falardeau (2003, p.680) de considérer la compréhension comme un travail d'objectivation au cours duquel, à partir d'éléments explicites et implicites du texte comme de connaissances partageables, «le lecteur cherche à construire un sens acceptable socialement" dans "une structure globalisante", et de définir l'interprétation comme «le point de vue d'une sujet (le lecteur) sur un objet (le 
texte) ", c'est-à-dire une recherche de signification extrinsèque, liée à la polysémie dudit texte et nécessitant un investissement subjectif de sa part, même si cette vision personnelle et donc singulière du texte nécessite de "passer par la confrontation sociale pour acquérir sa légitimité » (ibid., p. 675, 684). Ainsi avons-nous regroupé dans la catégorie $\mathrm{C}$ les réponses qui citent l'apprentissage de la compréhension sans l'associer à celui de l'interprétation, et également celles qui font référence à l'enseignement d'une "méthodologie» de la lecture ${ }^{17}$. Nous n'avons gardé dans la catégorie $\mathrm{F}$ que les réponses qui mentionnent explicitement l'apprentissage de l'interprétation des textes, qui évoquent leur polysémie (" débats ») ou la recherche de leur portée philosophique, mais aussi celles qui font référence à un sens ou une intention d'auteur à retrouver.

Tableau 4 : Types de réponses obtenues à la question Q6 du sondage

Q6 : Que signifie, selon vous, l'expression « lire un texte littéraire et se l'approprier »? (PRGM 2018 C2 et C3)

\begin{tabular}{|l|l|l|l|}
\hline A & Comprendre (associé à d'autres réponses) & $25 / 60$ & $42 \%$ \\
\hline B & Comprendre (réponse exclusive) & $25 / 60$ & $42 \%$ \\
\hline C & $\begin{array}{l}\text { S'immerger, entrer en empathie avec les personnages, éprouver des émotions } \\
\text { fictionnelles }\end{array}$ & $11 / 60$ & $18 \%$ \\
\hline D & Faire des liens avec d'autres œuvres & $11 / 60$ & $18 \%$ \\
\hline E & Faire des liens avec sa vie personnelle & $6 / 60$ & $10 \%$ \\
\hline F & Interpréter, donner du sens & $5 / 60$ & $8 \%$ \\
\hline G & Mettre des mots sur sa réception de l'œuvre, à parler de l'œuvre & $5 / 60$ & $8 \%$ \\
\hline H & Absence de réponse & $2 / 60$ & $3 \%$ \\
\hline
\end{tabular}

Pour la question Q6, relative à l'appropriation des textes, nous avons regroupé les réponses selon les activités mentales proposées et, parce que l'activité de compréhension est très majoritaire, nous avons dissocié les réponses où elle est évoquée seule de celles qui l'associent à une autre activité.

Tableau 5 : Types de réponses obtenues à la question Q4 du sondage

\begin{tabular}{|l|l|l|l|}
\hline \multicolumn{4}{|l|}{ Q4 : Quel sens donnez-vous à l'expression « sujet lecteur »? } \\
\hline A & Le sujet lecteur est un lecteur compétent & $19 / 60$ & $32 \%$ \\
\hline B & Le sujet lecteur est un lecteur impliqué & $11 / 60$ & $18 \%$ \\
\hline C & Le sujet lecteur est le lecteur considéré dans sa subjectivité & $11 / 60$ & $18 \%$ \\
\hline D & Le sujet lecteur est celui qui lit & $10 / 60$ & $17 \%$ \\
\hline E & Non-réponse & $7 / 60$ & $12 \%$ \\
\hline F & Autres & $2 / 60$ & $3 \%$ \\
\hline
\end{tabular}

31 Pour la classification des réponses à la question Q4, nous avons observé les mots qui caractérisent le sujet lecteur. Ceci nous a permis de faire quatre catégories : l'une (D) 
associée au verbe «lire» sans autre explication; une autre (B) aux notions d'implication et d'engagement; une troisième (A) aux verbes traduisant des activités intellectuelles (décoder, comprendre, analyser, interpréter, faire des hypothèses) ou une aptitude (travailler en autonomie); et la dernière (c) à des termes exprimant la subjectivité du lecteur (affects, vécu, goûts, empathie, être touché) ou sa singularité (bagage culturel).

Nous proposons maintenant d'analyser les résultats présentés, en énonçant quelques constats.

Constat $\mathrm{n}^{\circ} 1$ : Ces réponses laissent apparaitre une prédominance générale pour une conception culturelle de l'enseignement de la littérature.

Pour $77 \%$ des PES interrogés, la priorité est de donner accès au patrimoine littéraire (Q1A). «Faire découvrir aux élèves des œuvres et auteurs traditionnels et plus originaux", "construire une culture commune par la connaissance d'ouvrages", «mettre en réseau des œuvres pour constituer une culture littéraire chez l'élève » sont des exemples de réponses apportées. Cet objectif assigné à l'enseignement de la littérature n'a rien d'étonnant puisqu'il correspond aux exigences des programmes, qui préconisent, en $\mathrm{CM} 1$ et $\mathrm{CM} 2$, de faire lire sept ouvrages de littérature chaque année, de plus en plus longs et complexes, parmi lesquels deux œuvres du patrimoine (MEN, 2015) ou classiques (MEN, 2018) en CM1 et trois en CM2, le reste étant à choisir dans la littérature de jeunesse contemporaine. Par ailleurs, pour un tiers des PES, l'enseignement de la littérature doit donner des repères en termes de genres (Q1D). En revanche, pour l'appropriation des textes littéraires, l'établissement de liens avec d'autres œuvres n'est mentionné que par un PES sur cinq (Q6D). Ceci donne l'impression d'une représentation standardisée de la culture littéraire, loin du concept de bibliothèque intérieure théorisé par P. Bayard (2007, p. 74-75), comme étant «cet ensemble de livres [...] sur lequel toute personnalité se construit et qui organise ensuite son rapport aux textes et aux autres ", c'est-à-dire une bibliothèque mentale qui s'appuie, selon A. Rouxel (2013, p. 121), sur « le réseau de relations qu'un lecteur établit entre un texte et d'autres textes » et porte donc la marque de sa singularité de sujet.

Constat $n^{\circ} 2:$ La corrélation des réponses aux trois questions montre également que les PES ont une vision encore assez techniciste de l'enseignement de la littérature. Ainsi, constatons-nous que, pour un tiers d'entre eux, il y a équivalence entre « sujet lecteur » et lecteur compétent (Q4A) et que, parmi les enjeux de l'enseignement, ce sont les compétences de compréhension qui sont mises en avant (Q1C). Dans le même esprit, il faut noter que, pour définir l'action de s'approprier les textes, $84 \%$ des PES citent le processus de compréhension (Q6A et Q6B), et que la moitié d'entre eux se contente de cette définition (Q6B). Il apparait donc une forte tendance à valoriser dans la lecture littéraire ce qui relève de la compréhension et des méthodes liées aux stratégies de lecture, c'est-à-dire le travail du lectant. Ceci nous semble entrer en corrélation avec l'importance donnée à l'enseignement explicite de la compréhension dans les programmes actuels mais aussi sans doute dans les pratiques expertes auxquels les PES sont confrontés ${ }^{18}$. 

décalage entre les représentations que les PES se font de l'enseignement de la lecture littéraire et les préconisations des chercheurs en didactique de la littérature, dont les programmes scolaires portent la trace. Il apparait que, dans leurs représentations de la lecture littéraire et de l'appropriation des textes, les PES confèrent une fonction disproportionnée à la compréhension par rapport au rôle qu'ils attribuent aux affects et à l'expression de la subjectivité. Les injonctions ministérielles, en mettant particulièrement l'accent, depuis 2018, sur la compréhension explicite, en sont peutêtre la cause.

41 Nous allons regarder maintenant ce qu'il en est des pratiques de classe des PES, à travers les descriptions de séances d'enseignement rapportées dans les CR de visites.

\section{Les pratiques effectives pendant les séances de littérature en classe}

Avant d'observer ces comptes rendus, rappelons que les visites dont il est question sont faites, dans la classe du PES, par ses deux tuteurs: un PEMF en responsabilité d'une classe à l'année et un formateur de l'Éspé/Inspé ${ }^{19}$ appartenant soit à l'équipe dite «transversale» chargée du tronc commun de la formation, soit à une équipe disciplinaire spécialisée dans une des disciplines enseignées à l'école. Parmi les formateurs Éspé/Inspé se trouvent des enseignants aux statuts divers : ex-professeurs des écoles, ex-professeurs du second degré et enseignants-chercheurs. Aux visites des tuteurs s'ajoutent également celles des $\mathrm{CPC}^{20}$, chargés de venir en aide aux enseignants débutants de leur circonscription. Il découle de cette organisation que la plupart des formateurs qui rendent visite aux PES ne sont eux-mêmes pas spécialistes de didactique

Pratiques, $187-188 \mid 2020$ 
de la littérature. Selon leur statut, leur expérience passée, leur intérêt personnel, ils ont une connaissance plus ou moins précise et actualisée de cet enseignement.

Les stagiaires reçoivent en général sept visites dans l'année : trois du tuteur PEMF, deux du tuteur Éspé/Inspé et deux du CPC. Nous ne disposons pas de tous les CR pour les 144 PES concernés mais nous en avons en moyenne quatre ou cinq, majoritairement ceux des deux tuteurs.

4 Pour analyser les séances décrites dans les comptes rendus, nous avons observé trois éléments ${ }^{21}$ :

- les activités structurées, orales ou écrites, menées dans la séance ${ }^{22}$,

- les modalités de travail : cours dialogué, travail de groupe, travail individuel,

- l'approche des textes privilégiée : investissement ou distanciation.

Pe repérage des occurrences relatives à chaque rubrique, nous avons retenu les traits saillants de chaque séance, quand ils étaient notés dans le compte rendu. Étant donné qu'aucun compte rendu ne permet de saisir tous les aspects d'une séance et que certaines séances présentent différentes phases, avec des activités changeantes et sous des modalités évolutives, les chiffres obtenus ne donnent pas un panorama précis de toutes les séances décrites mais uniquement des tendances générales.

Les résultats obtenus sont présentés ci-dessous, les chiffres donnés indiquant le nombre d'occurrences observées parmi les 140 séances décrites et le pourcentage qui lui correspond, sachant donc que certaines séances peuvent correspondre à plusieurs rubriques :

Tableau 6 : Types d'activités menées dans la séance

\begin{tabular}{|c|c|c|c|}
\hline \multicolumn{4}{|c|}{ T1 : Activités menées } \\
\hline A & Réponses à un questionnaire individuel & 28 & $20 \%$ \\
\hline B & Repérage, tri, comparaison, résumé & 54 & $39 \%$ \\
\hline & Travaux d'écriture & 58 & \multirow{9}{*}{$41 \%$} \\
\hline & Écrits de travail (sans précision) & 20 & \\
\hline & Dessins de travail & 4 & \\
\hline & Écrits d'anticipation & 12 & \\
\hline C & Écrits réactifs & 5 & \\
\hline & Écrits en « je » fictif & 8 & \\
\hline & Écrits en « je » non-fictif & 2 & \\
\hline & Écrits dans le carnet de lecteur & 2 & \\
\hline & Écrits créatifs & 5 & \\
\hline \multirow{4}{*}{ D } & Activités orales structurées & 14 & \multirow{4}{*}{$10 \%$} \\
\hline & Débat & 2 & \\
\hline & Mise en voix & 9 & \\
\hline & Jeu de rôle & 3 & \\
\hline
\end{tabular}



nous pourrions considérer qu'ils relèvent tous de la catégorie des «écrits de travail » (Tauveron, 1999, p. 31) puisqu'ils sont au service de la lecture littéraire et n'ont pas de finalité pour eux-mêmes, mais nous les avons distingués selon ce qui en était dit dans le compte rendu. Les distinctions opérées concernent ainsi l'énonciation («je » fictif ou non fictif), le recours à la représentation imagée ( dessins de travail $\left.{ }^{23} »\right)$, le support utilisé (avec le carnet de lecteur) ou l'activité mise en jeu : «écrits réactifs 》 (affects, connotations ${ }^{24}$ ), « écrits d'anticipation » (compréhension de la logique du récit), «écrits créatifs " (jeu d'écriture sur le texte : imitation, contrainte, complément). Certaines rubriques peuvent donc se recouper. L'appellation «écrits de travail » a été maintenue uniquement pour ceux qui étaient nommés ainsi dans le compte rendu, sans autre précision.

Tableau 7 : Types de modalités de travail dans la séance

\begin{tabular}{|l|l|c|c|}
\hline \multicolumn{4}{|l|}{ T2 : Modalités de travail } \\
\hline A & Cours dialogué prof/élèves & 53 & $38 \%$ \\
\hline B & Travail individuel & 63 & $45 \%$ \\
\hline C & Travail de groupes ou binômes & 32 & $23 \%$ \\
\hline
\end{tabular}

Parmi les modalités de travail, nous appelons "cours dialogué ", l'échange collectif dans lequel l'enseignant pose des questions auxquelles un ou plusieurs élèves donnent les réponses, sans que l'organisation des propos soit anticipée autrement que comme une discussion collective gérée par le maitre.

Tableau 8 : Type d'approches des textes

\begin{tabular}{|c|c|c|c|}
\hline \multicolumn{4}{|c|}{ T3 : Approches des textes } \\
\hline A & Observer, comprendre, analyser, synthétiser & 124 & $89 \%$ \\
\hline B & Imaginer, s'immerger dans la fiction & 17 & $12 \%$ \\
\hline C & Donner ses impressions, parler de ses représentations, évoquer des connotations & 11 & $8 \%$ \\
\hline $\mathrm{D}$ & Exprimer ses goûts personnels ou un jugement moral & 8 & $6 \%$ \\
\hline E & Proposer une interprétation & 6 & $4 \%$ \\
\hline F & Réfléchir à l'acte de lire (métacognition) & 4 & $3 \%$ \\
\hline
\end{tabular}

Pour ce qui est de l'approche des textes, nous avons opté pour des rubriques relatives à l'engagement cognitif ou émotionnel attendu du lecteur dans l'activité proposée, selon qu'il impose une attitude distanciée ou impliquée. Nous pouvons donc distinguer tout ce qui relève de la mise à distance du texte, c'est-à-dire la compréhension et l'analyse, nécessitant une objectivation des informations recueillies (T3A) et tout ce qui renvoie à l'implication subjective, qu'il s'agisse de l'immersion dans la fiction et des phénomènes d'empathie (T3B), des réactions personnelles liées à la concrétisation imageante (T3C) 
ou à l'axiologie (T3D) mais également de l'engagement interprétatif (T3E). Dans la rubrique "proposer une interprétation » sont en effet rangées les rares séances pour lesquelles le $\mathrm{CR}$ mentionne explicitement les mots "interprétation » ou "débat interprétatif» mais aussi un travail de mise en voix proposé comme acte d'interprétation pour travailler la compréhension d'un texte.

51 Nous avons classé à part les activités réflexives sur l'activité lectorale, relevant de la métacognition qui, avec un score de $3 \%$, restent très marginales (T3F). Si ces activités exigent une posture distanciée, elles demandent aux élèves de reconnaitre les différentes instances lectrices activées en eux quand ils lisent, que ce soit l'intellect, l'imaginaire ou le corps ému.

52 Nous allons maintenant présenter quelques constats que nous pouvons faire au vu des résultats obtenus.

Constat $\mathrm{n}^{\circ} 5$ : Conformément aux recommandations explicitement énoncées dans les programmes (et répétées dans les cours de didactique), $41 \%$ des PES utilisent l'écriture pour faire travailler la littérature (T1C). On voit que, par leur nature, la moitié au moins de ces écrits sollicitent la subjectivité des élèves : écrits d'anticipation, écrits réactifs, écrits en « je » fictif ou non, écrits créatifs, écrits dans le carnet de lecteur. Il apparait cependant que le recours à l'écrit, pour mobiliser le lisant, n'est pas une habitude généralisée.

Constat $n^{\circ} 6$ : Le recours à des activités orales structurées, c'est-à-dire autres que les échanges collectifs gérés par l'enseignant ou ceux d'un travail de groupe, sont extrêmement rares. Mise en voix et jeux de rôle sont utilisés dans moins de 10 \% des séances. Par ailleurs, sur les sept occurrences d'un travail de mise en voix, l'une concerne des textes poétiques et quatre des textes théâtraux, ce qui signifie que la mise en voix n'est presque jamais utilisée pour lire des extraits de récits. Quant au débat, il n'apparait que très marginalement (deux occurrences).

Constat $n^{\circ} 7$ : Les interactions entre élèves sont assez peu favorisées dans les séances de littérature. Ainsi voit-on que $38 \%$ des séances sont conçues sous forme de cours dialogué (T2A), dans lequel le PE gère la parole à partir des quelques questions qu'il a préparées. De plus, dans $45 \%$ des cas, le travail demandé est un travail individuel (T2B) qui peut être ou non suivi d'une mise en commun ou d'une correction. Enfin, le travail en binôme ou en groupe n'est évoqué que pour 23 \% des séances (T2C). La communauté des lecteurs reste donc tributaire de l'enseignant pour dialoguer sur les textes. 
Constat $n^{\circ} 8$ : Dans une séance sur cinq parmi celles observées, soit $20 \%$, il est fait état d'un questionnaire auquel les élèves doivent répondre. Les limites desdits questionnaires ont pourtant été dénoncées (Tauveron, 2002, p. 88-93; Falardeau, 2003, p. 677; Bianco \& Lima, 2017, p. 91) et les programmes de 2015 encourageaient explicitement les enseignants à ne pas enfermer les élèves dans des questionnaires qui «baliseraient pour eux la lecture ». Il leur était, au contraire, demandé «d'apprendre aux élèves à questionner eux-mêmes les textes" (MEN, 2015 : 108). Cependant, le contenu des questionnaires proposés n'étant presque jamais décrit dans les comptes rendus de visite, il est difficile de tirer une conclusion de cette donnée : nous ne savons pas quels types d'opérations mentales est demandée aux élèves (prélever, inférer, interpréter, évaluer, s'interroger, etc.) et ne pouvons donc exclure que certains des questionnaires proposés par les PES soient élaborés de manière à solliciter la subjectivité du lecteur et/ou à laisser une large part à la réflexion et au questionnement autonome face au texte.

Constat $n^{\circ} 9$ : Les résultats obtenus mettent bien en évidence que la plupart des activités demandées aux élèves ont prioritairement pour but de favoriser la distanciation analytique et de développer les compétences de compréhension des textes. De tels objectifs apparaissent en effet dans $89 \%$ des séances décrites (T3A). La mise en jeu de la subjectivité par des activités favorisant l'implication du lecteur, que celle-ci passe par la mobilisation de son imaginaire (T3B), de ses émotions (T3C), de ses valeurs (T3D) ou d'un travail interprétatif (T3E), est présente seulement dans $30 \%$ des séances, soit moins d'une séance sur trois.

Nous proposons maintenant de revenir au questionnaire, pour voir si certaines représentations des difficultés de l'enseignement de la littérature ne permettent pas d'expliquer l'hiatus constaté entre les principes énoncés dans les instructions officielles (et dûment enseignés à l'Éspé) et les activités réellement menées en classe par les PES.

\section{Les représentations des difficultés}

Voici les résultats obtenus aux questions Q2 et Q3 portant sur le caractère facile ou non de l'enseignement de la littérature :

Tableau 9 : Types de réponses obtenues à la question Q2 du sondage

\begin{tabular}{|l|l|c|c|}
\hline \multicolumn{2}{|l|}{ Q2 : En quoi, selon vous, l'enseignement de la littérature est-il FACILE à mettre en œuvre ? } \\
\hline A & Engagement des élèves. Plaisir de lire qu'ils expriment. Adhésion facile à obtenir & $28 / 60$ & $47 \%$ \\
\hline B & Diversité et richesse des œuvres de LJ & $23 / 60$ & $38 \%$ \\
\hline C & Diversité des modalités de travail possibles & $12 / 60$ & $20 \%$ \\
\hline D & Diversité des supports pour les enseignants & $11 / 60$ & $18 \%$ \\
\hline
\end{tabular}




\begin{tabular}{|l|l|l|l|}
\hline E & Absence de réponse & $2 / 60$ & $3 \%$ \\
\hline
\end{tabular}

Tableau 10 : Types de réponses obtenues à la question Q3 du sondage

\begin{tabular}{|l|l|l|l|}
\hline \multicolumn{3}{|l}{ Q3 : En quoi, selon vous, l'enseignement de la littérature est-il DIFFICILE à mettre en œuvre ? } \\
\hline A & Difficultés didactiques (connaissances, temps) & $31 / 60$ & $52 \%$ \\
\hline B & Hétérogénéité des élèves & $22 / 60$ & $37 \%$ \\
\hline C & Difficultés à avoir une série d'ouvrages & $9 / 60$ & $15 \%$ \\
\hline D & Choix des œuvres & $7 / 60$ & $12 \%$ \\
\hline E & Absence de réponse & $2 / 60$ & $3 \%$ \\
\hline
\end{tabular}

Constat $\mathrm{n}^{\circ} 10$ : Parmi les difficultés énoncées, celle qui semble la plus prégnante est d'ordre didactique : il s'agit du manque de connaissances, corrélé au manque de temps pour préparer les séances. Cette difficulté est avancée par la moitié des stagiaires (Q3A). Cela corrobore le constat du faible nombre d'heures consacrées, pendant l'année de stage, à l'enseignement de la littérature ${ }^{25}$.

Constat $\mathrm{n}^{\circ} 11$ : Pour la moitié des PES, la littérature parait facile à enseigner, parce qu'elle suscite une forte adhésion des élèves qui prennent plaisir à la lecture des œuvres littéraires (Q2A). On peut ainsi se réjouir de voir de jeunes enseignants apprécier l'investissement affectif de leurs élèves dans le travail qu'ils leur proposent. Si le développement du plaisir de lire chez les élèves n'est un objectif affiché que par $36 \%$ des PES (Q1C), ils sont un peu plus nombreux à être ainsi sensibles à l'expression de cet enthousiasme et à ressentir son rôle de catalyseur pour l'enseignement de la littérature.

62 Mises en parallèle avec les constats effectués dans les classes, ces réponses positives font toutefois émerger un double paradoxe. C'est ce que nous allons voir dans le bilan.

\section{Bilan}

63 En mettent en tension les pratiques effectives des professeurs débutants avec leurs représentations de l'enseignement de la littérature et les injonctions institutionnelles, les constats effectués, présentent un bilan mitigé et paradoxal, dont nous allons faire état maintenant, en deux temps.

\section{Un bilan mitigé et paradoxal}

64 D'une part, l'analyse des données montrent que les travaux de Catherine Tauveron (1995;1999 2002) ont porté leurs fruits car les enseignants débutants ont bien saisi l'importance de la construction de compétences dans le domaine de la lecture littéraire, Par ailleurs, même si les PES ne sont majoritairement pas issus de filières de lettres, il apparait qu'ils sont sensibles à la diversité et la richesse des œuvres de littérature pour 
la jeunesse et se fixent comme enjeu prioritaire de développer et enrichir la culture littéraire de leurs élèves.

Cependant, il s'avère que la plupart d'entre eux semblent ne pas avoir saisi le virage épistémologique opéré en didactique de la littérature et qu'ils ont du mal à intégrer la notion de sujet lecteur dans leur conception de l'enseignement de la littérature. Comme on l'a vu, beaucoup réduisent les compétences de lecteur à des compétences de compréhension, négligeant ce qui relève de la capacité d'implication dans le texte, pourtant indispensable pour son appropriation. Si les professeurs d'école stagiaires restent sensibles au plaisir de lire exprimé par leurs élèves et disent souhaiter le développer, on observe, dans leurs pratiques de classe, peu d'activités mises en place pour laisser s'exprimer la singularité des réceptions des œuvres lues. Devant des élèves facilement prêts à participer aux échanges oraux du cours dialogué, les PES semblent ne pas ressentir de manière impérieuse la nécessité de prévoir des activités orales ou écrites accompagnées et articulées, faisant appel à subjectivité de chacun et favorisant le partage des impressions. C'est pourquoi nous parlons ici d'un premier paradoxe.

\section{Des perspectives pour la formation des PES}

Les résultats obtenus semblent indiquer quelques pistes pour la formation des enseignants en Inspé, selon deux axes.

Le premier axe concerne les modules de formation initiale des étudiants du master MEEF $1^{\text {er }}$ degré, dans lesquels les liens entre dimension cognitive et dimension sensible de l'approche des textes devraient sans doute être mieux démontrés afin de promouvoir des démarches mettant en jeu conjointement lisant et lectant, sans les opposer et sans hiérarchiser leurs rôles dans l'activité lectorale. Le travail sur la compréhension étant fortement valorisé par les programmes et dans les actions de formation proposées actuellement par les rectorats, il semble en effet important de rappeler que l'appropriation des textes littéraires nécessite la mobilisation du lecteur dans toutes ses dimensions, intellectuelle, émotionnelle, sensorielle et expérientielle.

68 Une telle prise de conscience passe par la mise en œuvre de dispositifs facilitant la lecture subjective et le partage des réceptions singulières, comme la mise en voix, le débat interprétatif, les cercles de lecture ou bien, à l'écrit, le journal de lecteur et le journal de personnage. Elle nécessite également une réflexion sur les effets ressentis en tant que sujet lecteur pendant cette expérimentation. Si de telles mises en œuvre sont assez chronophages en formation d'enseignants, elles s'avèrent cependant fructueuses (Larrivé, 2020).

69 Le deuxième axe concerne également la didactique, mais avec une orientation plus théorique, relative à la métacognition. Il nous semble important d'engager avec les professeurs en formation une réflexion sur le plaisir de lire, afin de lutter contre des représentations réductrices qui font obstacle au développement et à la mise en œuvre effective de dispositifs innovants. C'est donc sur la question du plaisir de lire que nous conclurons. 


\section{« Lectures de plaisir » et plaisirs de lire}

Dans le questionnaire proposé, nous avions à dessein demandé aux PES quel sens ils donnaient au terme « lectures de plaisir » utilisé dans les programmes de 2018. Voici leurs réponses :

Tableau 11 : Types de réponses obtenues à la question Q5 du sondage

\begin{tabular}{|l|l|l|l|}
\hline \multicolumn{4}{|c|}{ Q5 : Sens de l'expression « lecture de plaisir » } \\
\hline A & Lecture sans travail scolaire & $26 / 60$ & $43 \%$ \\
\hline B & Lecture libre, choisie & $19 / 60$ & $32 \%$ \\
\hline C & Lecture « pour le plaisir » & $10 / 60$ & $17 \%$ \\
\hline D & Lecture avec émotion & $2 / 60$ & $3 \%$ \\
\hline E & Absence de réponse & $0 / 60$ & $0 \%$ \\
\hline
\end{tabular}

71 Il apparait que l'immense majorité des PES, trois sur quatre, a bien saisi le sens de l'expression en considérant que la lecture de plaisir est une lecture non liée à un travail scolaire (Q5A) ou une lecture libre et choisie (Q5B). Dans les programmes de 2018, cette expression, utilisée au pluriel, est en effet synonyme de " lectures personnelles »:

Les lectures personnelles ou lectures de plaisir sont encouragées sur le temps scolaire, elles sont choisies librement : les élèves empruntent régulièrement des livres qui correspondent à leurs intérêts et à leurs projets. (MEN, 2018 : 13).

S'il est tout à fait justifié que les programmes de cycle 3 demandent aux enseignants de promouvoir les lectures personnelles pour développer chez leurs élèves des habitudes de lecteur, l'expression « lectures de plaisir », officialisée par les instructions officielles, nous semble cristalliser un second paradoxe, plus fondamental et contre-productif dans notre discipline. Par ce choix terminologique, le texte des programmes oppose en effet les lectures personnelles ne demandant aucun travail et procurant du plaisir, aux lectures scolaires dont on devine implicitement, par contraste, qu'elles nécessitent un travail mais ne génèrent pas de plaisir. Il est peu probable que cette représentation de la lecture littéraire scolaire permette aux enseignants débutants de comprendre ce qui se joue dans la séance de littérature en termes de construction de la personnalité de l'élève lecteur par la mise en jeu de sa subjectivité.

En prenant en compte les multiples dimensions de l'activité lectorale, il apparait pourtant que le plaisir de lecteur est de nature protéiforme. Plaisir de l'immersion fictionnelle et plaisir de faire vivre des personnages, plaisir esthétique d'observer une forme textuelle et plaisir d'y chercher du sens, plaisir de partager ses impressions avec ses pairs et plaisir de répondre ensemble aux questions que l'on se pose, plaisir de se sentir lecteur autonome et plaisir de comprendre ce qui se passe en soi quand on lit, etc., toute une gamme de bonheurs de lire, bien connus des grands lecteurs, s'offre aux élèves. En prendre conscience permet à l'enseignant d'opter pour des dispositifs de lecture qui mobilisent le lecteur dans sa diversité et lui donnent l'occasion d'expérimenter ces plaisirs variés et complémentaires de la lecture. 


\section{BIBLIOGRAPHIE}

BAYARD, P. (2007). Comment parler des livres que l'on n'a pas lus ?. Paris : Éditions de Minuit.

BIANCO, M. \& LIMA, L. (2017). Comment enseigner la compréhension en lecture ?. Paris : Hatier.

BRILLANT-RANOU, N. et al. (dirs) (2020). Un dictionnaire de didactique de la littérature. Paris :

H. Champion.

DUFAYS, J.-L., GEMENNE, L., LEDUR, D. (2005) [1996]. Pour une lecture littéraire. Louvain-la-Neuve : De

Boeck.

FALARDEAU, E. (2003). «Compréhension et interprétation : deux composantes complémentaires de la lecture littéraire ». Revue des sciences de l'éducation 29 (3), p. 673-694. En ligne : https:// www.erudit.org/fr/revues/rse/2003-v29-n3-rse966/011409ar/.

JoUVE, V. (1992). L'Effet personnage dans le roman. Paris : Presses universitaires de France.

LANGLADE, G. (2007). « La lecture subjective ». Québec français 145, p. 71-73. En ligne : https://

www.erudit.org/fr/revues/qf/2007-n145-qf1178006/47315ac/.

LARRIVÉ, V. (2015). « Empathie fictionnelle et écriture en “je” fictif ». Repères. recherches en didactiqu du français langue maternelle 51, p. 157-176. En ligne : https://journals.openedition.org/ reperes/913.

LARRIVÉ, V. (2020, à paraître). « Le journal de lecteur comme dispositif de formation à la lecture sensible pour les professeurs de français stagiaires $»$. In : Actes du colloque des $20^{e s}$ rencontres des chercheurs en didactique de la littérature. 12-14 juin 2019 Rennes.

Ministère de l'Éducation nationale, de l'Enseignement supérieur et de la Recherche (2016). Le Carnet de lecteur. Paris : Éduscol. En ligne : http://langage.ac-creteil.fr/IMG/pdf/ carnet_de_lecteur.pdf.

Ministère de l'Éducation nationale, de la Jeunesse et des Sports (2015). « Programme pour le cycle 3 ». Bulletin officiel spécial $n^{\circ} 11$ du 26 novembre 2015. En ligne : http://eppee.ouvaton.org/IMG/pdf/ programmes_cycle_3._bo_spe_11_26-11-2015_504351.pdf.

Ministère de l'Éducation nationale, de la Jeunesse et des Sports (2018). « Programme des enseignements du cycle $3 »$. Bulletin officiel $n^{\circ} 30$ du 26 juillet 2018. En ligne : https:// cache.media.education.gouv.fr/file/30/05/0/ensel169_annexe2V2_986050.pdf.

PICARD, M. (1986). La Lecture comme jeu. Paris : Éditions de Minuit.

ROUXEL, A. (2013) « L'avènement du sujet lecteur. Avancées scientifiques et perspectives pour l'enseignement de la littérature ». In : Ahr, S. \& Joole, p. (dirs). Carnet/journal de lecteur/lecture. Quels usages pour quels enjeux, de l'école à l'université ?. Namur : Presses universitaires de Namur, p. 115-128.

SHAWKY-MILCENT, B. (2016). La Lecture, ça ne sert à rien! Usages de la littérature au lycée et partout ailleurs... Paris : Presses universitaires de France.

SZAC M. \& DUVIVIER J.-M. (2006). Le feuilleton d'Hermès. Paris : Bayard.

TAUVERON, C. (1995). Le Personnage. Une clef pour la didactique du récit à l'école élémentaire. Neuchâtel : Delachaux \& Niestlé. 
TAUVERON, C. (1999). «Comprendre et interpréter le littéraire à l'école : du texte réticent au texte proliférant ». Repères. Recherches en didactique du français langue maternelle 19, p. 9-38. En ligne. https://www.persee.fr/doc/reper_1157-1330_1999_num_19_1_2289.

TAUVERON, C. (dir.) (2002). Lire la littérature à l'école. Pourquoi et comment conduire cet apprentissage spécifique? de la GS au CM2. Paris : Hatier

\title{
ANNEXES
}

\section{Questionnaire donné en version papier aux PES de l'académie de Toulouse en mars 2019}

\author{
Recto \\ MERCI de bien vouloir remplir le RECTO avant de tourner la feuille pour répondre aux questions au VERSO. \\ PES en formation à l'ÉSPÉ de \\ enseignant en cycle \\ niveau de \\ classe \\ PES inscrit en M2 PFPA1 PFPA2 (entourer la bonne réponse) \\ dans le groupe \\ (pour les Toulousain-e-s) \\ Que signifie, selon vous, enseigner la littérature ? \\ En quoi, selon vous, l'enseignement de la littérature est-il facile à mettre en œuvre ? \\ En quoi, selon vous, l'enseignement de la littérature est-il difficile à mettre en œuvre ? \\ Verso

Quel sens donnez-vous à l'expression « sujet lecteur » ?
Comment comprenez-vous l'expression « lecture de plaisir » (PRGM 2018 C2 et C3) ?
Que signifie, selon vous, l'expression « lire un texte littéraire et se l'approprier » (PRGM 2018
C3)?
Cette année, faites-vous un mémoire ou un écrit réflexif?
Quel que soit votre écrit scientifique, indiquez dans quel domaine de recherche vous travaillez:
MERCI pour votre participation

\section{NOTES}

1. Éspé : École Supérieure du Professorat et de l'Éducation. Les établissements publics du supérieur chargés de la formation des enseignants ont été appelés Éspé de septembre 2014 à septembre 2019.

2. MEN : Ministère de l'Éducation Nationale. Cette contraction sera utilisée pour le Ministère de l'Éducation nationale, de l'Enseignement supérieur et de la Recherche ainsi que pour le Ministère de l'Éducation nationale, de la Jeunesse et des Sports.

3. Inspé : Institut National Supérieur du Professorat et de l'Éducation. Les Éspé ont été transformées en Inspé depuis septembre 2019.

4. En 2015, la liste était complétée par le mot « blogs » qui disparait en 2018. 
5. De septembre 2014 à juillet 2019, cette alternance s'est faite, pour les professeurs des écoles stagiaires de Toulouse, sur une périodicité correspondant à la semaine d'école, avec deux jours et demi passés en classe et deux jours et demi à l'Éspé.

6. Dans la période qui nous intéresse, chaque PES toulousain était associé à un titulaire, directeur déchargé ou collègue à mi-temps, dont il prenait la classe en charge pendant la moitié du temps d'école. Les deux enseignants, titulaire et stagiaire, devaient donc négocier la répartition des disciplines à enseigner et c'est ainsi que certains stagiaires n'ont eu à assurer aucune séance de littérature pendant toute l'année car leur binôme préférait garder la responsabilité de cet enseignement.

7. Mathématiques, Français, EPS, Histoire, Géographie, EMC, SVT, Technologie, Physique, Arts plastiques, Musique.

8. Master MEEF : Master Métiers de l'Enseignement, de l'Éducation et de la Formation

9. Une enquête interne de la mention « Professorat des écoles » de l'Éspé de Toulouse montre qu'en 2016, $25 \%$ des étudiants M1 du MEEF $1^{\text {er }}$ degré venaient d'un cursus de Lettres, $32 \%$ des sciences humaines, $26 \%$ d'une formation scientifique, $9 \%$ des filières DEG (droit, économie et gestion) et $3 \%$ d'une licence professionnelle ( $5 \%$ n'avaient pas renseigné le questionnaire). La moitié d'entre eux sont devenus stagiaires dès leur entrée en M2 et plus du quart dans les deux années suivantes.

10. On peut compter entre dix et quatorze heures pour la didactique de la littérature sur les trois cycles de l'école primaire.

11. Les dossiers récoltés pour l'année 2017-2018 sont malheureusement trop incomplets pour être utilisés, car ils ne contiennent souvent que les CR de visite du premier semestre.

12. C'est souvent ainsi que les temps de récitation sont nommés dans les rapports de visite.

13. Szac \& Duvivier (2006).

14. Exercices extraits notamment des fiches Je lis, je comprends du manuel d'entrainement Cléo CM2. Français (Éd. Retz, 2011, p. 8-37) comportant 30 pages d'exercices de compréhension explicite ou des propositions de Lector \& Lectrix, ouvrage de S. Cèbe et R. Goigoux (Éd. Retz, 2009) à l'attention des enseignants de CM, $6^{2 \text { ème }}$ et Segpa. Voir à l'adresse suivante: https://www.ac-orleans-tours.fr/pedagogie/ ecole_1er_degre/ressources_chateauroux/je_lis_je_comprends/.

15. Il ne s'agit donc pas d'un QCM.

16. Le questionnaire est reproduit en annexe.

17. Méthodologie de lecture que nous avons donc associée aux stratégies de compréhension.

18. Pendant leur année de formation, les PES font plusieurs stages d'observation et de pratique accompagnée (Sopa) dans les classes de professeurs des écoles maitres formateurs (PEMF), titulaires d'un Certificat d'aptitude aux fonctions d'instituteur ou de professeur des écoles maître formateur (Cafipemf, équivalent, pour le primaire, du Certificat d'aptitude aux fonctions de formateur académique [Caffa] obtenu par des professeurs du secondaire).

19. Les mêmes principes ont été reconduits depuis la rentrée 2019, date à laquelle les Éspé ont changé de nom.

20. CPC : conseillers pédagogiques de circonscription. 
21. Nous avons également noté le genre de texte abordé (théâtre, poésie, récit) et le mode de première réception du texte (lecture faite en amont de la séance ou pendant, lecture oralisée par le maitre ou par les élèves, lecture silencieuse) mais nous ne traiterons pas ces données ici.

22. Par "activités structurées ", nous entendons les activités prévues en amont et organisées minutieusement par le PES.

23. Nous avons intégré dans les « dessins de travail » la représentation d'un personnage ou d'un lieu de la fiction.

24. Quatre des cinq occurrences d' "écrits réactifs » concernent l'écriture de mots clés demandés après la lecture d'un texte littéraire, la quatrième concerne la description des images mentales (visuelles, sonores ou sensorielles) advenues à la lecture d'une description.

25. Comme les heures de formation sont principalement concentrées au premier semestre, on peut estimer qu'en mars, époque du sondage, il ne reste au mieux qu'une dizaine d'heures d'enseignement en didactique du français et donc très peu en littérature. Il est donc probable que, si les mêmes questions étaient posées en fin d'année universitaire, elles recevraient des réponses assez similaires.

\section{RÉSUMÉS}

Dans cet article nous nous intéressons aux pratiques effectives d'étude des textes observées, en cycle 3, dans les classes des professeurs des écoles stagiaires. Sachant que les programmes en cours demandent explicitement de prendre en compte la subjectivité des élèves dans la réception des textes littéraires, l'objectif est de voir comment cette injonction se traduit en termes d'activités proposées et d'outils utilisés dans les séances de littérature. L'expression et le partage des impressions personnelles, liées aux émotions, aux goûts, aux conceptions, à l'expérience et aux souvenirs de chacun, sont-ils effectivement sollicités dans les activités écrites ou orales mises en place?

Cette analyse s'appuie sur l'observation des comptes rendus de visites élaborés pendant deux ans par les formateurs qui assurent la fonction de tuteurs. Un sondage effectué auprès des stagiaires permet également de confronter les résultats de ces observations aux représentations que les stagiaires se font de la lecture littéraire et du plaisir de lire, pour tenter d'identifier les forces de résistance en jeu dans les conceptions de la didactique de la littérature.

This article is about current habits for study of literary texts in elementary school, observed in the classes of trainee teachers practicing in $4^{\text {th }}-5^{\text {th }}$ grades/Years 6-7. Knowing that school curricula explicitly request to take into account the subjectivity of students in the reception of literary texts, the objective is to see how this order is followed in terms of activities and tools during literature lessons. Does the expression and the sharing of personal impressions, linked to the emotions, tastes, conceptions, experience and memories of each one, take place within the written or oral activities implemented?

This analysis is based on the observation of visit reports drawn up over two years by trainers acting as tutors. A survey has also been carried out, what makes possible to compare the results 
of these observations with the trainee teachers conceptions of literary reading and reading pleasure and, thereby, allows to identify some resistances at play in understanding didactics of literature.

INDEX

Keywords : Reader's subjectivity, Theorie of reading, Literature didactics, Literature lessons, Teachers training, Elementary school

Mots-clés : subjectivité du lecteur, théorie de la lecture, didactique de la littérature, séances d'enseignement de la littérature, formation des enseignants, école élémentaire

\section{AUTEUR \\ VÉRONIQUE LARRIVÉ}

Université de Toulouse-Jean Jaurès, LLA-Créatis, F-31058 Toulouse, France 